\title{
Desflurane and neural control of airway tone
}

\author{
Charles W. Emala, MD
}

Published online: 25 August 2011

(C) Canadian Anesthesiologists' Society 2011

The study by Myers et al. ${ }^{1}$ in this edition of the Journal adds to our understanding of the role of desflurane in reactive airway disease. The authors compare the ability of sevoflurane with that of desflurane to alter respiratory mechanics following a cholinergic contraction in normal or sensitized rabbits whose lungs were ventilated through a tracheostomy. This study is an important addition to a growing body of literature which addresses an important clinical question: Does the choice of volatile anesthetic matter in patients with reactive airway disease, and more specifically, is desflurane a bad choice in patients with reactive airway disease?

The airway is exposed to two primary irritants during general anesthesia with a volatile anesthetic, i.e., a foreign body (endotracheal tube or laryngeal mask airway device [LMAD]) acting as a mechanical irritant and a volatile anesthetic which can elicit a chemical irritation. C-fibres, including excitatory non-adrenergic non-cholinergic (eNANC) nerves, innervate the upper airway and are activated by transient receptor potential (TRP) channels to release tachykinins which can elicit smooth muscle contraction. Desflurane has been shown to activate TRP A1 receptors on C-fibres, inducing a tachykinin-mediated airway response. ${ }^{2,3}$ A secondary important neural reflex pathway which responds to airway irritation is the cholinergic pathway whereby parasympathetic nerve fibres travelling within the vagus nerve release acetylcholine which induces airway smooth muscle contraction. The current study was designed to evaluate the effect of desflurane on the neurotransmitter of this latter neural

C. W. Emala, MD ( $\square)$

Department of Anesthesiology, Columbia University, 622 West 168th St., P\&S Box 46, New York, NY 10032, USA

e-mail: cwe5@columbia.edu pathway, namely, acetylcholine. The authors demonstrate that desflurane is comparable with sevoflurane in its ability to attenuate bronchoconstriction induced by activation of muscarinic receptors on airway smooth muscle by intravenous methacholine. These results are consistent with previous studies which implicated TRP channels on C-fibres as the culprit of desflurane-induced ${ }^{2,3}$ bronchoconstriction observed in clinical studies. ${ }^{4,5}$ Although not a topic of the current study, desflurane activation of C-fibres would also account for the frequently observed clinical complication of cough. ${ }^{6,7}$

In isolated human bronchi, desflurane was equally potent as halothane and isoflurane at relaxing proximal but not distal bronchi. ${ }^{8}$ This finding is also consistent with the present study since neural innervation is thought to be primarily cholinergic in large central airways and eNANC in peripheral airways. In previous studies of isolated airways $^{9-12}$ or tracheostomized and ventilated lungs of animals ${ }^{13}$ from multiple species, desflurane was shown to be at least as effective as other volatile anesthetics in directly relaxing airway smooth muscle. Taken together, these studies, which are performed in the absence of intact innervation, suggest that clinical difficulties with desflurane and the airway are not likely related to direct airway smooth muscle effects but are mediated by differential effects on irritant neural reflexes.

The current study is an important addition to our mechanistic understanding of differential effects of volatile anesthetics on bronchoconstriction. This study demonstrates that sevoflurane and desflurane are similar in their abilities to prevent bronchoconstriction of cholinergic origin occurring in sensitized airways. It should be emphasized that desflurane was delivered to an intubated trachea $^{11,13}$ (or isolated airway tissue) in this and previous 
animal studies, ${ }^{8-10,12}$ thus sparing the upper airway (upper trachea, oropharynx, nasopharynx) from direct topical exposure to the volatile anesthetic. During mask or LMAD anesthesia, there is an additional opportunity for desflurane to activate TRP channels in C-fibres and elicit cough. This detrimental effect of desflurane on neural reflexes as opposed to airway smooth muscle is further supported by the clinical experience with desflurane where coughing/laryngospasm is reported far more often than bronchoconstriction. $^{4-7}$

This elegant in vivo animal study provides further clarity to show that reported clinical difficulties with desflurane do not involve cholinergic reflexes but likely involve desflurane activation of $\mathrm{C}$-fibres resulting in cough/laryngospasm and reduced bronchodilation.

\section{Le desflurane et le contrôle neuronal du tonus des voies aériennes}

L'étude de Myers et coll., ${ }^{1}$ présentée dans ce numéro du Journal, offre de nouvelles perspectives dans notre compréhension du rôle du desflurane dans l'hyperréactivité des voies aériennes. Les auteurs comparent la capacité du sévoflurane à celle du desflurane à modifier la mécanique respiratoire suite à une contraction cholinergique chez des lapins normaux et sensibilisés dont les poumons ont été ventilés via trachéostomie. Cette étude est un ajout important à un corpus grandissant de littérature qui traite d'une question clinique cruciale : le choix d'anesthésique volatil est-il important chez les patients souffrant d'une hyperréactivité des voies aériennes? Et, plus spécifiquement, le desflurane est-il un mauvais choix chez de tels patients?

Pendant une anesthésie générale réalisée à l'aide d'un anesthésique volatil, les voies aériennes sont exposées à deux principaux agents irritants, soit un corps étranger (sonde endotrachéale ou masque laryngé [LMAD]), lequel agit comme un irritant mécanique, et un anesthésique volatil, qui peut provoquer une irritation chimique. Les fibres $\mathrm{C}$, y compris les nerfs excitateurs non adrénergiques non cholinergiques (eNANC), innervent les voies aériennes supérieures et sont activées par les canaux TRP (transient receptor potential) afin de libérer des tachykinines qui peuvent provoquer une contraction des muscles lisses. Il a été démontré que le desflurane activait les récepteurs TRP A1 sur les fibres $C$, induisant ainsi une réaction des voies aériennes médiée par la tachykinine. ${ }^{2,3} \mathrm{La}$ voie cholinergique est une voie secondaire importante de réflexe neuronal qui réagit à une irritation des voies aériennes; dans cette voie, les fibres nerveuses parasympathiques du nerf vague libèrent de l'acétylcholine, laquelle induit la contraction des muscles lisses des voies aériennes. L'étude présentée ici a été conçue de façon à évaluer l'effet du desflurane sur le neurotransmetteur de cette deuxième voie neuronale, soit l'acétylcholine. Les auteurs démontrent que le desflurane est comparable au sévoflurane quant à sa capacité à atténuer la bronchoconstriction induite par l'activation des récepteurs muscariniques sur les muscles lisses des voies aériennes par la méthacholine intraveineuse. Ces résultats concordent avec ceux des études précédentes, selon lesquelles les canaux TRP sur les fibres C étaient responsables de la bronchoconstriction induite par le desflurane ${ }^{2,3}$ observée dans les études cliniques. ${ }^{4,5}$ Bien que ce point ne soit pas traité dans l'étude en question ici, l'activation par le desflurane des fibres $\mathrm{C}$ pourrait également être responsable d'une complication clinique fréquemment observée : la toux. ${ }^{6,7}$

Dans une étude réalisée sur des bronches humaines isolées, le desflurane s'est montré aussi puissant que l'halothane et l'isoflurane pour relaxer les bronches proximales, mais pas les bronches distales. ${ }^{8}$ Cette découverte concorde avec la présente étude, étant donné qu'on pense que l'innervation neuronale est principalement cholinergique dans les voies aériennes centrales importantes et relève des eNANC dans les voies aériennes périphériques. Dans les études précédentes portant sur des voies aériennes isolées ${ }^{9-12}$ ou des poumons trachéostomisés et ventilés d'animaux ${ }^{13}$ de plusieurs espèces, il a été démontré que le desflurane était au moins aussi efficace que les autres agents anesthésiques volatils pour relaxer directement les muscles lisses des voies aériennes. Ensemble, ces études réalisées en l'absence d'une innervation intacte suggèrent que les difficultés cliniques rencontrées lorsque le desflurane entre en contact avec les voies aériennes ne sont probablement pas liées aux effets directs sur les muscles lisses des voies aériennes, mais qu'elles sont plutôt médiées par des effets différentiels sur les réflexes neuronaux irritants.

L'étude en question ici contribue de façon importante à notre compréhension des mécanismes des effets différentiels des anesthésiques volatils sur la bronchoconstriction. Cette étude démontre que le sévoflurane et le desflurane sont semblables dans leurs capacités à prévenir la bronchoconstriction d'origine cholinergique survenant dans le cas de voies aériennes sensibilisées. Il convient de souligner que le desflurane est distribué dans une trachée intubée $^{11,13}$ (ou un tissu d'une voie aérienne isolée) dans cette étude animale et les précédentes, ${ }^{8-10,12}$ ce qui épargne les voies aériennes supérieures (trachée supérieure, oropharynx, nasopharynx) d'une exposition directe à l'anesthésique volatil. Lors d'une anesthésie au masque ou au masque laryngé, le desflurane a une occasion supplémentaire d'activer les canaux TRP dans les fibres C et 
de provoquer la toux. Cet effet nuisible du desflurane sur les réflexes neuronaux plutôt que sur les muscles lisses des voies aériennes est en outre appuyé par notre expérience clinique avec le desflurane; en effet, la toux et les laryngospasmes sont rapportés bien plus souvent que la bronchoconstriction. ${ }^{4-7}$

Cette étude animale in vivo sophistiquée met en lumière le fait que les difficultés cliniques rapportées avec le desflurane ne sont pas liées aux réflexes cholinergiques, mais qu'elles sont probablement liées à l'activation par le desflurane des fibres C, laquelle entraîne la toux / le laryngospasme et une bronchodilatation réduite.

Competing interests None declared.

\section{References}

1. Carole F. Myers CF, Fontao F, Janosi TZ, Boda K, Petak F, Habre $W$. Sevoflurane and desflurane protect cholinergic-induced bronchoconstriction of hyperreactive airways in rabbits. Can J Anesth 2011; 58. DOI:10.1007/s12630-011-9578-3

2. Satoh JI, Yamakage M, Kobayashi T, Tohse N, Watanabe H, Namiki A. Desflurane but not sevoflurane can increase lung resistance via tachykinin pathways. Br J Anaesth 2009; 102: 704-13.

3. Satoh J, Yamakage M. Desflurane induces airway contraction mainly by activating transient receptor potential A1 of sensory C-fibers. J Anesth 2009; 23: 620-3.
4. Goff MJ, Arain SR, Ficke DJ, Uhrich TD, Ebert TJ. Absence of bronchodilation during desflurane anesthesia: a comparison to sevoflurane and thiopental. Anesthesiology 2000; 93: 404-8.

5. von Ungern-Sternberg BS, Saudan S, Petak F, Hantos Z, Habre $W$. Desflurane but not sevoflurane impairs airway and respiratory tissue mechanics in children with susceptible airways. Anesthesiology 2008; 108: 216-24.

6. Zwass MS, Fisher DM, Welborn LG, et al. Induction and maintenance characteristics of anesthesia with desflurane and nitrous oxide in infants and children. Anesthesiology 1992; 76: 373-8.

7. Klock PA Jr, Czeslick EG, Klafta JM, Ovassapian A, Moss J. The effect of sevoflurane and desflurane on upper airway reactivity. Anesthesiology 2001; 94: 963-7.

8. Mercier FJ, Naline E, Bardou M, et al. Relaxation of proximal and distal isolated human bronchi by halothane, isoflurane and desflurane. Eur Respir J 2002; 20: 286-92.

9. Mazzeo AJ, Cheng EY, Bosnjak ZJ, Coon RL, Kampine JP. Differential effects of desflurane and halothane on peripheral airway smooth muscle. Br J Anaesth 1996; 76: 841-6.

10. Park KW, Dai HB, Lowenstein E, Sellke FW. Epithelial dependence of the bronchodilatory effect of sevoflurane and desflurane in rat distal bronchi. Anesth Analg 1998; 86: 646-51.

11. Lele E, Petak F, Fontao F, Morel DR, Habre W. Protective effects of volatile agents against acetylcholine-induced bronchoconstriction in isolated perfused rat lungs. Acta Anaesthesiol Scand 2006; 50: 1145-51.

12. Wiklund $C U$, Lindsten $U$, Lim $S$, Lindahl SG. Interactions of volatile anesthetics with cholinergic, tachykinin, and leukotriene mechanisms in isolated Guinea pig bronchial smooth muscle. Anesth Analg 2002; 95: 1650-5.

13. Habre W, Petak F, Sly PD, Hantos Z, Morel DR. Protective effects of volatile agents against methacholine-induced bronchoconstriction in rats. Anesthesiology 2001; 94: 348-53. 